\title{
UMLAUFENDES WEIDEN IN BRACHIARIA BRIZANTHA CV. MARANDU ZUR VERBESSERUNG DER MILCHPRODUKTION
}

\section{ORIGINALER ARTIKEL}

SOARES, Marcio Groto ${ }^{1}$, CAMARGO, Simone Cristina², FRASSETTO, Matheus Orlandin ${ }^{3}$, ABREU, Hélio Mar de ${ }^{4}$

SOARES, Marcio Groto. Et al. Umlaufendes Weiden in Brachiaria brizantha cv. Marandu zur Verbesserung der Milchproduktion. Revista Científica Multidisciplinar Núcleo do Conhecimento. Jahr. 06, Hrsg. 09, Vol. 07, S. 104-118. September 2021. ISSN: 2448-0959, Zugangslink: https://www.nucleodoconhecimento.com.br/veterinaria-de/umlaufendes-weiden, DOI: 10.32749/nucleodoconhecimento.com.br/veterinaria-de/umlaufendes-weiden

\section{ZUSAMMENFASSUNG}

In Molkereibetrieben ist es für eine Gewinnsteigerung erforderlich, die Effizienz der Produktionsfaktoren, insbesondere der Ernährung, zu erhöhen, was sich auf die Fixkosten auswirkt. Zunehmend wird ein Gleichgewicht zwischen Futter- und Weideangebot angestrebt. Die Rotationsweidemethode wurde von mehreren Herstellern übernommen, da sie eine gute Produktivität auf einer kleineren Fläche bietet. Allerdings haben die Weiden, die bei dieser Art der Beweidung verwendet werden, trotz ihrer hervorragenden Qualität einen höheren Nährstoffbedarf. Das Ziel dieser Arbeit war eine wirtschaftliche Analyse der Kosten der Milchproduktion durch die Einführung von Umtriebsweiden und den Austausch von einheimischem Weideland gegen Brachiaria brizantha $c v$. marandu, auf einem Familienbauernhof. Diese Arbeit wurde in der Stadt Foz do Iguaçu vorbereitet. Es wurde eine Fläche von

\footnotetext{
${ }^{1}$ Absolvent einer Universität.

2 Doktortitel.

${ }^{3}$ Lehrer.

${ }^{4}$ Absolvent einer Universität.
}

RC: 99523

Verfügbar in: https://www.nucleodoconhecimento.com.br/veterinariade/umlaufendes-weiden 
$6300 \mathrm{~m}^{2}$ genutzt, die mit einem Elektrozaun in 28 Paddocks von $225 \mathrm{~m}^{2}$ aufgeteilt wurde. Die Gesamtproduktionskosten betrugen 6.047,40 R $\$$ mit einer positiven Marge von 5.143,61 R\$ pro Jahr. Nach den Ergebnissen der wirtschaftlichen Analysen wird der Schluss gezogen, dass die Einpflanzung von Umtriebsweiden und der Austausch von heimischem Weideland gegen Brachiaria brizantha cv. Marandu ist ein tragfähiges Management, da die Einnahmen aus dem Verkauf von Milch ausreichten, um die Gesamtkosten der Produktion zu decken.

Stichworte: Kosten, Milch, Futter, Streikposten, Handling.

\section{EINLEITUNG}

Brasilien belegt den vierten Platz in der Weltrangliste der Milchproduktion. Im Jahr 2018 entfielen 34\% der Gesamtproduktion des Landes auf die Region Süd (ANUÁRIO LEITE, 2019). Allein der Bundesstaat Paraná produzierte in diesem Jahr durchschnittlich 4,37 Milliarden Liter Milch, wofür westliche Paraná 19\% verantwortlich war (IBGE, 2018).

Milchvieh im Bundesstaat Paraná ist eine typische Tätigkeit der Familienbetriebe, die etwa $85,1 \%$ der staatlichen Produzenten ausmacht (SEAB, 2018). Die meisten dieser Produzenten nutzen Weideland als Hauptnahrungsquelle für Tiere. Die durchschnittliche Fläche mit Weiden im Staat beträgt 16,6 Hektar (IPARDES, 2009).

Obwohl Brasilien in der Milchproduktion hervorsticht, liegt seine Produktivität bei rund 1.689 I / Kuh / Jahr, was niedriger ist als in den Vereinigten Staaten, China, Russland, Argentinien, der Europäischen Union und Neuseeland, die mehr als 3 I / Kuh / Jahr produzieren. Einer der Faktoren, die für die geringe Milchleistung verantwortlich sind, ist die Verwendung von schlecht produktiven Gräsern mit geringer Nährstoffqualität sowie eine unzureichende Bewirtschaftung von Tieren und Weiden (GONÇALVES et al., 2003).

RC: 99523

Verfügbar in: https://www.nucleodoconhecimento.com.br/veterinariade/umlaufendes-weiden 
Eine Möglichkeit, die Tierproduktion in Weidesystemen zu intensivieren, ist die Einführung von verbessertem Futter, das besser an das Klima angepasst ist, mit höherem Produktionspotenzial und besserer Qualität (JANK, 2017). Unter den verschiedenen In Brasilien existierenden Futtermitteln sticht die Hybridsorte Brachiaria brizantha $c v$. marandu Gras hervor, für einen guten Nährwert und eine hohe Biomasseproduktion, die gute Anpassung an Böden mit mittlerer und guter Fruchtbarkeit, die Toleranz gegenüber Bodensäure (FONSECA et al., 2010).

Die richtige Bewirtschaftung der Weide sollte ein weiterer Punkt sein, der bei den Eigenschaften von Milchrindern zu beachten ist. Eine der Beweidungen, die sich als lebensfähig erwiesen hat, ist die Rotation, bei der die Weide in drei oder mehr Streikposten unterteilt wird. Diese Art der Beweidung hat mehrere Vorteile, wie zum Beispiel: größere Weidegleichmäßigkeit, größere Nutzung von Futter, höhere Besatzrate und längere Langlebigkeit von Unkräutern, die Klumpen bilden (FUKUMOTO et al., 2010).

Verschiedene Studien haben gute Ergebnisse für Milchkühe gezeigt, mit der Implantation von Rotationsweiden in Brachiaria brizantha cv. marandu Gras (GONÇALVES, 2003); (DEMSKI, 2013), (MOURA, 2017). Laut (ANDRADE, 2008) verträgt diese Art von Weide keine kontinuierliche Beweidung.

Mit der richtigen Wahl des Futters, der richtigen Verwaltung und der besseren Nutzung der Grundstücksfläche versucht es, Einsparungen für den Produzenten zu erzielen, da es die Produktionskosten senkt. Gemäß (GERON, 2012) sind die Produktionskosten die Summe aller Beträge, die für die Summe und die Vorgänge und Dienstleistungen ausgegeben werden, die im Produktionsprozess einer bestimmten Aktivität verwendet werden. Mit der Kostenuntersuchung lässt sich überprüfen, wie die in einem Produktionsprozess eingesetzten Ressourcen vergütet werden und wie die Wirtschaftlichkeit der Tätigkeit ist (GERON et al., 2014).

RC: 99523

Verfügbar in: https://www.nucleodoconhecimento.com.br/veterinariade/umlaufendes-weiden 
Das Ziel dieser Arbeit war es daher, die wirtschaftliche Analyse der Kosten der Milchproduktion durch die Implementierung von Rotationsweiden und den Austausch von einheimischer Weide gegen Weide von Brachiaria brizantha cv. marandu durchzuführen, auf einem bäuerlichen Familienanwesen.

\section{MATERIAL UND METHODEN}

Das Experiment wurde in einem Milchviehbetrieb in der Stadt Foz do Iguaçu - PR, Breitengrad: -25.5469, Längengrad: -54.5882 25 32' 49" Süd, 54 35' 18" West durchgeführt. Dieses Anwesen umfasst eine Fläche von 5,0 Hektar, in diesem Bereich wurde eine traditionelle Milchwirtschaft in der Region entwickelt, bei der die Tiere Zugang zur gesamten Weidefläche hatten, bestehend aus einheimischem Gras mit geringer Produktivität, es gab keine Verfügbarkeit von Mineralsalz, für laktierende Tiere stand nur Kochsalz im Trog und nur $10 \mathrm{~kg}$ Melkergänzung zur Verfügung und der Zugang zu Wasser erfolgte über einen Bach, der das Grundstück durchfließt.

Das Anwesen verfügt über sechs Milchkühe, bestehend aus vier erwachsenen Kühen, zwei laktierenden Kühen und zwei getrockneten Kühen sowie zwei Färsen.Diese Tiere wurden das ganze Jahr über auf der gesamten Weidefläche bewirtschaftet und die laktierenden Tiere erhielten $10 \mathrm{~kg}$ industrialisiertes Futter pro Tag.

Die Arbeit begann mit einem Besuch der Immobilie für eine Diagnose. Die Messungen der Weidefläche wurden mittels GPS (Abbildung 1) und auch mit Bodensammlung (Abbildung 2) durchgeführt. Die Ergebnisse der Bodenanalyse sind (Tabelle 1).

RC: 99523

Verfügbar in: https://www.nucleodoconhecimento.com.br/veterinaria- 
Abbildung 1 - Satellitenfoto des Versuchsgebiets mit der Abgrenzung der Weidefläche:

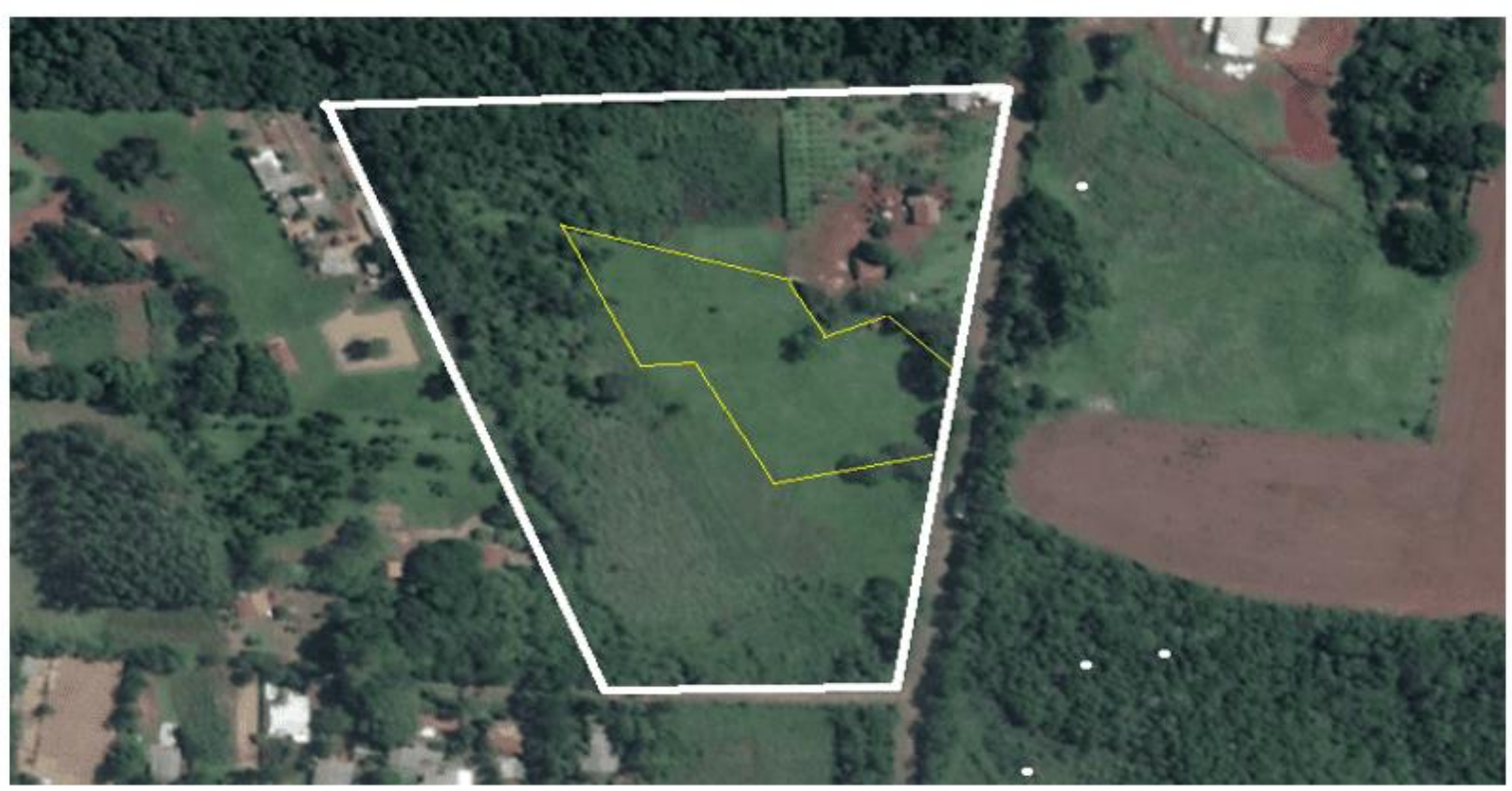

Quelle: Google Earth.

Abbildung 2 - Bodenprobenentnahme:

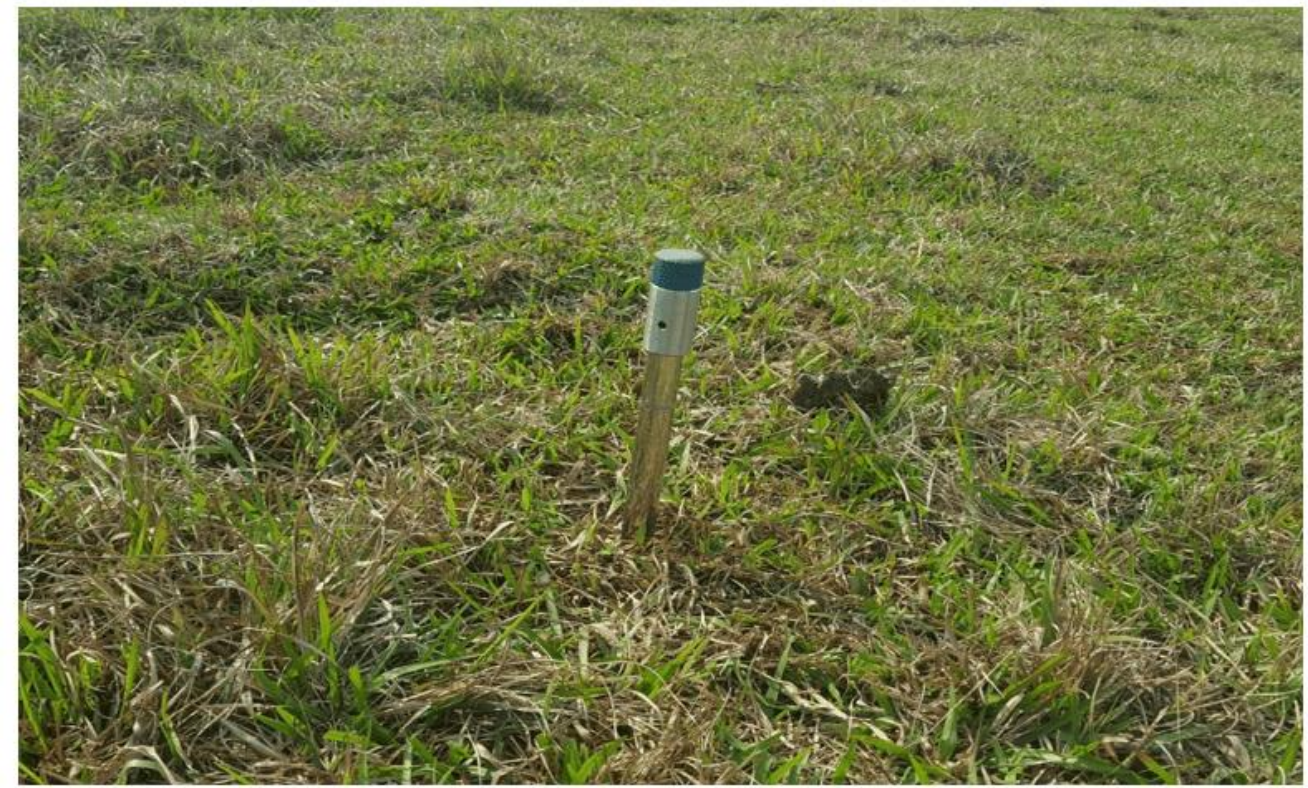

Quelle: Persönliches Archiv.

RC: 99523

Verfügbar in: https://www.nucleodoconhecimento.com.br/veterinariade/umlaufendes-weiden 
Tabelle 1 - Ergebnisse der Bodenanalyse des Versuchsgebiets:

\begin{tabular}{|c|c|c|c|c|c|c|c|c|c|c|c|c|c|c|}
\hline $\mathrm{pH}$ & & & $\mathrm{K}$ & $\mathrm{Ca}$ & $\mathrm{Mg}$ & $\mathrm{Al}$ & $\mathrm{H}+\mathrm{Al}$ & CTC & SB & $\mathrm{V}$ & $\mathrm{m}$ & $\mathrm{Ca}$ & $\mathrm{Mg}$ & $\mathrm{K}$ \\
\hline \multirow[t]{2}{*}{$\mathrm{CaCl} 2$} & M.O & $P($ res) & & & & & & & & & & & & \\
\hline & $\mathrm{g} / \mathrm{Kg}$ & $\mathrm{mg} / \mathrm{dm} 3$ & \multicolumn{5}{|c|}{$\mathrm{mmol} / \mathrm{dm} 3$} & $\%$ & & & \multicolumn{4}{|c|}{$\%$ СТС } \\
\hline 5,2 & 38 & $\overline{14}$ & 3 & 21 & 5 & 2,7 & 29 & 58 & 29 & 50 & 8 & 36 & 9 & 5 \\
\hline
\end{tabular}

Quelle: FZEA/USP.

Nach Erhalt der Bodenanalyse wurden Bodenkorrekturberechnungen durchgeführt, wobei der V\% $58 \%$ betrug. Um den pH-Wert des Bodens zu korrigieren, waren 800 $\mathrm{kg} \mathrm{ha}^{-1}$ dolomitischer Kalkstein mit 86\% PRNT erforderlich. Um den Phosphor zu korrigieren, war es notwendig, $250 \mathrm{~kg} \mathrm{ha}^{-1}$ einfachen Superphosphat-Dünger zuzugeben. Für die Produktionsdüngung wurden $250 \mathrm{~kg}$ ha-1 landwirtschaftlicher Harnstoff verwendet (FRASSETO, 2015).

Um mit der Bodenvorbereitung zu beginnen, wurde die Anwendung von Kalkstein im gesamten Versuchsgebiet angewendet, und bald darauf wurde ein schweres Gitter mit einer Tiefe von $40 \mathrm{~cm}$ verwendet, das dazu diente, Kalkstein in den Boden einzubauen, Oberflächenverdichtungen zu brechen und Bedingungen für das Wachstum der neuen Futterarten zu geben.

Für eine Dimensionierung der für die Beweidung erforderlichen Fläche wurden die Kühe mit einer Genauigkeit von 95\% durch ein Wägeband gewogen, insgesamt $1.950 \mathrm{~kg}$ oder 4,5 UA. Aufgrund der Tatsache, dass Milchkühe verwendet wurden, wurde festgelegt, dass die Tiere am Ende eines Weidetages Zugang zu einem neuen Streikposten haben würden. Daher wurden Daten von (FRASSETO 2015) verwendet, bei denen eine UA (450 kg Lebendgewicht) mindestens $50 \mathrm{~m}^{2}$ pro Tag Weidefläche benötigt. Da man wusste, dass es 4,5 AE gab, betrug die Fläche jedes Streikpostens $225 \mathrm{~m}^{2}$. Insgesamt wurden 28 Streikposten auf einer Fläche von 0,63 Hektar errichtet. Während des Baus der Streikposten war es notwendig, drei Korridore mit einer Breite von $2 \mathrm{~m}$ hinzuzufügen.

RC: 99523

Verfügbar in: https://www.nucleodoconhecimento.com.br/veterinariade/umlaufendes-weiden 
Für die Spaltung der Streikposten wurden 50 Mourões, 72 Bewehrungsstäbe von 10 $\mathrm{mm}, 32$ Bewehrungsstäbe von $12 \mathrm{~mm}, 500 \mathrm{~m}$ dreiverzinkter Draht für etwa den gesamten Umfang der Fläche und $2500 \mathrm{~m}$ Elektrokunststoffdraht für die interne Aufteilung der Streikposten, 150 Isolatoren vom braunen Typ, 22 Hakenisolatoren und 114 Isolatoren vom Typ Bewehrung verwendet. Draht mit einer Höhe von $80 \mathrm{~cm}$ wurde in Bezug auf den Boden verwendet und alle $10 \mathrm{~m}$ wurde ein 1,5 m Bewehrungsstahl mit Isolator implantiert, um die Höhe im Verhältnis zum Boden zu halten, elektroplastischer Draht wurde auch verwendet, um die Streikposten zu teilen und die Formationskosten zu reduzieren. Zur Elektrifizierung des Drahtes wurde ein Sentinel-Elektrifier Modell 30.000 mit mindestens 5000 Volt verwendet.

Abbildung 3 - Skizze der Streikpostenteilung:

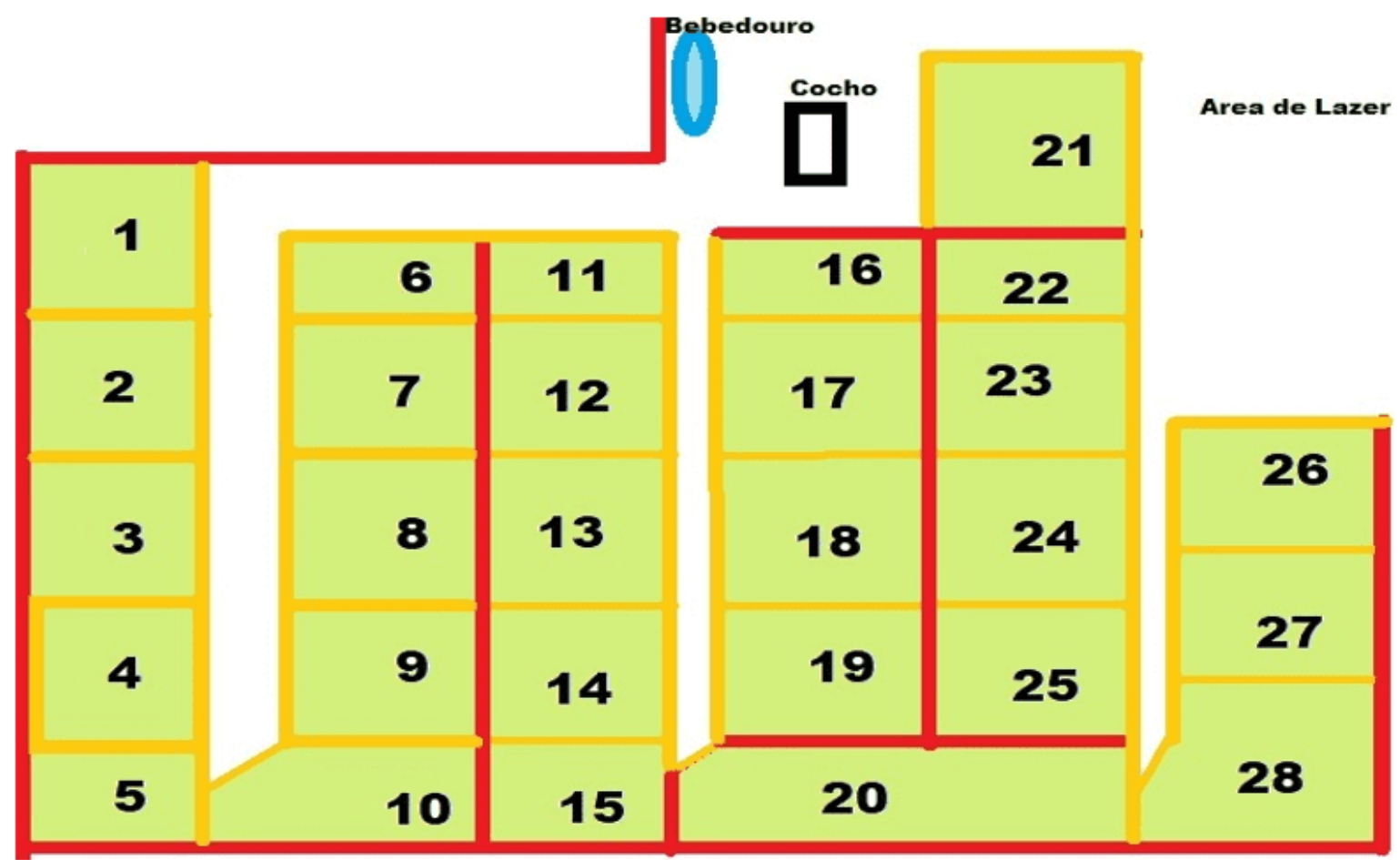

Quelle: Persönliches Archiv.

Das gewählte Futter war Brachiaria Brizantha cv. marandu, die Bewirtschaftung dieses Futters beginnt, wenn die Pflanze $30 \mathrm{~cm}$ am Eingang und $15 \mathrm{~cm}$ am Ausgang

RC: 99523

Verfügbar in: https://www.nucleodoconhecimento.com.br/veterinariade/umlaufendes-weiden 
Andrade erreicht (2008); Fonseca (2010). Am 15.11.2019 wurde mit der Aussaat von Grundfutter und der Pflanzdüngung begonnen, mit der Aussaatmethode und anschließender Einarbeitung von Saatgut und einfachem Phosphatdünger. Die Samen wurden mit $15 \mathrm{~kg} \mathrm{ha}^{-1}$ inkrustiert.

Die erste Düngung der Produktion wurde 60 Tage nach der Pflanzung durchgeführt, wobei $125 \mathrm{~kg} \mathrm{ha}^{-1}$ landwirtschaftlicher Harnstoff mit 45\% Stickstoff ausgebracht wurden. Die erste Beweidung begann am 03.02.2020, 77 Tage nach der Pflanzung. Die zweite Produktionsdüngung, die vor $125 \mathrm{~kg}^{-1}$ von landwirtschaftlichem Harnstoff angewendet wurde, wurde beim ersten Weidezyklus durchgeführt, der 28 Tage nach Beginn der ersten Beweidung war.

Für die Ruhe der Tiere wurde eine Wassertränke mit ca. $100 \mathrm{~L}$ Wasser hinzugefügt, Mineralsalz für Milchkühe wurde hinzugefügt und die eigenen Bäume des Grundstücks spendeten Schatten.

Um die wirtschaftliche Analyse und Bewertung der Kosten der Milchproduktion durchzuführen, identifizierten wir die beteiligten Akteure sowie die Werte, die die Auswirkungen der Durchführung des Projekts auf diese Agenten darstellen, auch unter Berücksichtigung des Abschlusses eines Jahres.

Um die Kosten für die Umsetzung des Projekts zu bewerten, berücksichtigten wir die Kosten für Investitionen in die Bodenvorbereitung und die Pflanzung des Grases sowie Investitionen in Infrastruktur und Ausrüstung bei der Bildung von Streikposten.

Um die Produktionskosten zu bewerten, verwendeten wir die von Matsunaga (1976) vorgeschlagene Struktur der Betriebskosten der Produktion. In denen die effektiven Betriebskosten (COE) den Ausgaben des Produzenten für ein Jahr mit dem Kauf von Futtermitteln, den Ausgaben für die Instandhaltung von Weideland und Medikamenten entsprechen.

RC: 99523

Verfügbar in: https://www.nucleodoconhecimento.com.br/veterinariade/umlaufendes-weiden 
Für die Gesamtproduktionskosten (CTP) wurden Abschreibungen zu (COE) hinzugerechnet. Die jährliche Abschreibung der Weide wurde durch die Summe der für den Bau des Elektrozauns notwendigen Vorräte über einen Zeitraum von zehn Jahren berechnet. Abschreibung von 10\% pro Jahr (CANZIANI et al., 2000).

Zur Berechnung der Einnahmen wurde der Nettoumsatz berücksichtigt, d. b. der betrag, der bei der Milchlieferung pro Jahr abzüglich der Gesamtproduktionskosten erzielt wird (MARTIN, 1997).

\section{ERGEBNISSE UND DISKUSSION}

Innerhalb der landwirtschaftlichen Produktion ist es notwendig, eine Planung durch den Produzenten zu haben, damit es Wachstum und Entwicklung der Aktivität gibt. Daher besteht der erste Schritt bei der Untersuchung der Machbarkeit eines Projekts darin, die Kosten für seine Umsetzung zu erfragen. Die Kosten für die Durchführung dieses Projekts, von der Vorbereitung des Bodens für die Pflanzung der Weide, bis zur Bildung der Streikposten beliefen sich auf $\mathrm{R} \$ 3.023,70$.

Vor der Implantation von Gras B. brizantha cv. marandu, es war notwendig, den Boden vorzubereiten. Investitionen im Zusammenhang mit der Bodenbearbeitung sind in (Tabelle 2) beschrieben.

Tabelle 2 - Investitionskosten für Bodenvorbereitung und -pflanzung:

\begin{tabular}{|l|l|l|l|}
\hline Beschreibung & Einheit & Menge & Wert (R\$) \\
\hline Bodenanalyse & Uni & 1 & 33,00 \\
\hline $\begin{array}{l}\text { Dolomitkalkstein (PRNT } \\
\text { 86\%) }\end{array}$ & $\mathrm{Kg}$ & 800 & 156,00 \\
\hline Superphosphat (19.00.00) & $\mathrm{Kg}$ & 250 & 287,46 \\
\hline Traktor-Abrationszeit & $\mathrm{H}$ & 2 & 400,00 \\
\hline Verkrusteter Samen & $\mathrm{Kg}$ & 15 & 287,01 \\
\hline
\end{tabular}

RC: 99523

Verfügbar in: https://www.nucleodoconhecimento.com.br/veterinariade/umlaufendes-weiden 


\begin{tabular}{|l|l|l|l|}
\hline $\begin{array}{l}\text { Landwirtschaftliche } \\
\text { Harnstoff }\end{array}$ & $\mathrm{Kg}$ & 250 & 400,00 \\
\hline Herbizid Glyphosat & $\mathrm{Ml}$ & 500 & 35,00 \\
\hline Öl 2T & $\mathrm{Ml}$ & 250 & 20,00 \\
\hline Kraftstoff - Benzin & $\mathrm{L}$ & 5 & 20,00 \\
\hline gesamt & & & $\mathbf{1 . 6 3 8 , 4 7}$ \\
\hline
\end{tabular}

Quelle: Projektdaten.

Die Kosten für Bodenbearbeitung und -bepflanzung machten $54,20 \%$ der Gesamtkosten für die Weideimplantation aus. Korrekturmaßnahmen und Bodendüngung sind nur ein Teil der Anforderungen, die für den Erfolg des Weideproduktionssystems erforderlich sind (PEREIRA et al., 2018).

Für den Produzenten ist es wichtig, zu investieren und eine gute Bodenvorbereitung und eine korrekte Umsetzung der Weide durchzuführen, immer auf der Suche nach einem Gleichgewicht im Boden-Pflanze-Tier-System (PEREIRA et al., 2018). Diese Managements werden die Steigerung der Tierproduktion sowie die Senkung der Futterkosten für Tierfutter beeinflussen.

Auf der Suche nach einer korrekten Bewirtschaftung der Weide wurde sie in Streikposten unterteilt. Investitionen in Infrastruktur und Ausrüstung bei der Bildung von Streikposten sind in (Tabelle 3) dargestellt.

Tabelle 3 - Kostentabelle für die Bildung von Streikposten mit dem Elektrozaun:

\begin{tabular}{|c|c|c|c|}
\hline Beschreibung & Einheit & Menge & Wert $(\mathrm{R} \$)$ \\
\hline Eletrix Draht & $\mathrm{m}$ & 500 & 184,00 \\
\hline $\begin{array}{l}\text { Elektroplastischer Draht } 500 \\
\text { m }\end{array}$ & $\mathrm{m}$ & 5 & 104,82 \\
\hline Brauner Isolator & Uni & 150 & 176,98 \\
\hline
\end{tabular}

RC: 99523

Verfügbar in: https://www.nucleodoconhecimento.com.br/veterinariade/umlaufendes-weiden 


\begin{tabular}{|c|c|c|c|}
\hline Bewehrungsisolator & Uni & 114 & 131,65 \\
\hline $\begin{array}{l}\text { Isolator vom Typ Kurzer } \\
\text { Haken }\end{array}$ & Uni & 20 & 24,00 \\
\hline Langer Hakenisolator & Eins & 2 & 4,60 \\
\hline Schalter & Eins & 3 & 36,00 \\
\hline Bewehrungsstahl (3/8") 1,5 m & Eins & 72 & 284,30 \\
\hline Bewehrungsstahl (1/2") 1,5 m & Eins & 2 & 186,18 \\
\hline PVC-Rohr 20 mm 6 m & Eins & 1 & 12,80 \\
\hline Digitales Voltmeter & Eins & 1 & 139,90 \\
\hline Elektrifikator & Eins & 1 & 100,00 \\
\hline gesamt & & & $1.385,23$ \\
\hline
\end{tabular}

Quelle: Projektdaten.

Die Kosten für Infrastruktur und Ausrüstung für die Bildung von Streikposten machen $45,80 \%$ der Gesamtkosten für die Umsetzung der Weide aus. Eine rationelle Futterbewirtschaftung hat die Vorteile der Weideaufteilung umfassend demonstriert, bei der eine größere Gleichförmigkeit der Weide, eine stärkere Nutzung von Futter, eine höhere Besatzdichte und eine längere Lebensdauer von verklumpenden Gräsern erreicht werden (FUKUMOTO, 2010), was im Fall von BB. brizantha cv. marandu. der Fall ist Lebenslauf. Der Einsatz des Elektrozauns in seinen unterschiedlichen Formen beim Streikposten ist ein notwendiges Werkzeug, um die für die Rotationsweide erforderliche Unterteilung mit geringen Kosten realisieren zu können (EMBRAPA, 1999).

Die Vermögenswerte, aus denen das Unternehmen besteht, unterliegen ständigen Abwertungen, hauptsächlich aufgrund von Verschleiß und Alterung. (Tabelle 4) enthält die Abschreibungsdaten des Elektrozauns.

RC: 99523

Verfügbar in: https://www.nucleodoconhecimento.com.br/veterinariade/umlaufendes-weiden 
Tabelle 4 - Abschreibungen auf Materialien und Ausrüstungen:

\begin{tabular}{|l|l|l|l|}
\hline Beschreibung & Gesamtbetrag R $\$$ & Haltbarkeit (Jahre) & Jährlicher Mietwert \\
\hline Elektrozaunbau & $1.385,25$ & 10 & 138,52 \\
\hline
\end{tabular}

Quelle: Projektdaten.

Tabelle 5 - Investitionszahlung:

\begin{tabular}{|l|l|l|l|}
\hline Beschreibung & $\begin{array}{l}\text { Gesamtbetrag } \\
\mathrm{R} \$\end{array}$ & $\begin{array}{l}\text { Zahlungszeit } \\
\text { (Jahre) }\end{array}$ & $\begin{array}{l}\text { Jährlicher } \\
\text { Mietwert }\end{array}$ \\
\hline Bereitstellungskosten & $3,023.70$ & $\mathbf{1 0}$ & 302.37 \\
\hline
\end{tabular}

Quelle: Projektdaten.

Um eine bessere jährliche Kontrolle über die Unternehmensführung $\mathrm{zu}$ haben, wurden die Investitionen für die Bereitstellung in 10 Jahre aufgeteilt. 4,20\% der jährlichen Einnahmen aus dem Verkauf von Milch, beschrieben in (Tabelle 5).

Um die Geldaufnahme in Form von Geld in einem Jahr zu berechnen, wurde der Betrag berücksichtigt, der dem Erzeuger pro Liter Milch gezahlt wurde, der täglich und direkt vom Formproduzenten für ein Jahr verkauft wurde. Zur Berechnung der Jahresmilchmenge wurde ein tägliches Melken am Morgen mit insgesamt 10 Litern/Tag betrachtet (Tabelle 6).

Tabelle 6 - Einnahmen aus dem Verkauf von Jahresmilch:

\begin{tabular}{|l|l|}
\hline Variablen & Gesamtwert \\
\hline Preis pro Liter (R\$) & 2,00 \\
\hline Insgesamt erzeugte Liter Milch (L) & $3.600,00$ \\
& \\
\hline
\end{tabular}

RC: 99523

Verfügbar in: https://www.nucleodoconhecimento.com.br/veterinariade/umlaufendes-weiden 
Endumsatz (R\$)

$7.200,00$

Quelle: Projektdaten.

Jedes Jahr muss der Produzent Geldausgaben tätigen, die als effektive Betriebskosten (COE) gelten. Berücksichtigt wurden die Kosten für Futtermittel, Aufwendungen für die Pflege von Weideland und Medikamente (Tabelle 7).

Tabelle 7 - Effektive Betriebskosten - COE, pro Jahr:

\begin{tabular}{|l|l|l|l|l|}
\hline Beschreibung & Einheit & Quant & $\begin{array}{l}\text { Einheitswert } \\
\mathrm{R} \$\end{array}$ & $\begin{array}{l}\text { Gesamtbetrag } \\
\mathrm{R} \$\end{array}$ \\
\hline Ration & $\mathrm{Kg}$ & 720 & 1,32 & 950,04 \\
\hline Landwirtschaftliche Harnstoff & $\mathrm{Kg}$ & 250 & 1,60 & 400,00 \\
\hline Elektrische Energie & $\mathrm{R} \$$ & 12 & 0,60 & 216,00 \\
\hline Brucellose-Impfstoff & Dosis & 6 & 1,62 & 9,72 \\
\hline Tollwutimpfstoff & Dosis & 6 & 1,48 & 8,88 \\
\hline Dectomax 50 ml & Uni. & 1 & 21,90 & 21,90 \\
\hline $\begin{array}{l}\text { Maul- und Klauenseuche- } \\
\text { Impfstoff }\end{array}$ & Dosis & 6 & 1,50 & 9,00 \\
\hline gesamt & & & & \\
\hline
\end{tabular}

Quelle: Projektdaten.

Es wird in dieser Arbeit beobachtet, dass die größten Ausgaben für die Produktion für Futtermittel getätigt werden. Laut (MARTINEZ, 2009) machen die Ausgaben für Ernährung durchschnittlich 67\% der Milchproduktionskosten in Brasilien aus. Daher werden Strategien gesucht, um die Verwendung von Diäten und anderen Nahrungsergänzungsmitteln zu reduzieren. Die Verbesserung der Weide und deren Bewirtschaftung ist eine Alternative, die dazu beiträgt, dass die Milchproduktion

RC: 99523

Verfügbar in: https://www.nucleodoconhecimento.com.br/veterinariade/umlaufendes-weiden 
wirtschaftlich rentabel ist. Aus diesem Grund ist eine Rotationsbeweidung zunehmend indiziert (MARION et al., 2010).

Rosestolato (2015) stellte bei der Analyse der Wirtschaftlichkeit der Milcherzeugung von zwei unterschiedlichen Eigenschaften in Bezug auf das technologische Produktionssystem fest, dass die (COE) auf dem Grundstück, auf dem die Kühe in einem kontinuierlichen Weidesystem gehalten und mit Kraftfutter und Mineralsalz ergänzt wurden, höher war als in einem Rotationsweidesystem und mit gehacktem Zuckerrohr ergänzt. Kraftfutter und Mineralsalz.

In der in dieser Studie bewerteten Eigenschaft wurden $10 \mathrm{~kg}$ industrialisiertes Futter pro stillendem Tier gegeben und nach der Durchführung des Projekts gab es eine Abnahme von $9 \mathrm{~kg}$, die den Tieren nur $1 \mathrm{~kg}$ Futter bot, wodurch die (COE) verringert wurde, wie von Rosestolato (2015) beobachtet. Die Ergänzung der Tiere auch bei der Änderung des Weidesystems ist jedoch wichtig, da sie hilft, bei Tieren die Nährstoffe zu liefern, die in der Zusammensetzung des Futters fehlen. Die Kosten für das Futter machten 44,70\% aller Produktionskosten aus.

Unter Berücksichtigung der Abschreibungen und der effektiven Betriebskosten (COE) wurden die Gesamtproduktionskosten in einem Jahr ermittelt (Tabelle 8).

Tabelle 8 - Gesamtproduktionskosten - CTP (Jahresbasis):

\begin{tabular}{|l|l|}
\hline Beschreibung & Gesamtbetrag R\$ \\
\hline Abschreibung & 138,52 \\
\hline COE-Kosten & $1.615,50$ \\
\hline Investitionszahlung & 302.37 \\
\hline gesamt & $\mathbf{2 . 0 5 6 , 3 9}$ \\
\hline
\end{tabular}

Quelle: Projektdaten.

RC: 99523

Verfügbar in: https://www.nucleodoconhecimento.com.br/veterinaria- 
In Ländern mit niedrigen Milchpreisen können die Erzeuger die Produktionskosten senken, indem sie die Beteiligung von Weiden an der Ernährung von Milchkühen erhöhen. In dieser Studie machten die Gesamtproduktionskosten 28,55\% des Jahreseinkommens aus.

Zur Berechnung der Einnahmen wurde der Nettoumsatz berücksichtigt, d. b. der durch die Milchlieferung pro Jahr erzielte Wert abzüglich der Gesamtproduktionskosten (Tabelle 9).

Tabelle 9 - Einnahmen (Jahresbasis):

\begin{tabular}{|c|c|c|}
\hline \multicolumn{2}{|l|}{ Beschreibung } & Gesamtbetrag $\mathrm{R} \$$ \\
\hline Einnahme & & $7.200,00$ \\
\hline $\begin{array}{l}\text { Gesamtproduktionskosten } \\
\text { Jahresbasis) }\end{array}$ & (CTP- & $2.056,39$ \\
\hline Gleichgewicht & & $5.143,61$ \\
\hline
\end{tabular}

Quelle: Projektdaten.

Laut Oliveira (2007) ist das Wissen um die kurzfristigen Auswirkungen der effektiven Betriebskosten (COE) für das Management des Unternehmens von grundlegender Bedeutung, und die Bruttomarge muss positiv sein, wenn negativ, wird die Unterbrechung der Produktion empfohlen.

Die Finanzanalyse der Durchführung dieses Projekts erwies sich als durchführbar (Tabelle 9). Angesichts der Analysen, die aus den erhaltenen Daten gewonnen wurden, wurde festgestellt, dass die Investition in die Rotationsweidetechnik bei Milchkühen eine wirtschaftlich tragfähige Rendite auf das investierte Kapital darstellt und dass der gleiche positive Einfluss auf die Produktion diese Aktivität wettbewerbsfähiger in diesem Sektor macht. So ermöglicht diese Technik die potenzielle Expression für die Milchproduktion und die Steigerung der Rentabilität der

RC: 99523

Verfügbar in: https://www.nucleodoconhecimento.com.br/veterinariade/umlaufendes-weiden 
Erzeuger, was die Milchtätigkeit auch in Zeiten des Milchpreisverfalls profitabel machen würde.

\section{ENDGÜLTIGE ÜBERLEGUNGEN}

Nach den Ergebnissen der wirtschaftlichen Analysen wird der Schluss gezogen, dass die Durchführung der Rotationsbeweidung und der Austausch von einheimischen Weiden durch Brachiaria brizantha cv. marandu ist ein lebensfähiges Management, da die Einnahmen aus dem Verkauf von Milch ausreichten, um die Gesamtkosten der Produktion zu decken.

Dieses semi-intensive Produktionssystem wird der lokalen Realität empfohlen, weil es eine Alternative zur technologischen Innovation ist, die in der Lage ist, die Kosten durch den Kauf von Futtermitteln zu senken und eine gute Tierleistung zu bieten, von der Änderung des Weidemanagements und der Einfügung von Gras mit besserem Nährwert und höherer Massenproduktion.

\section{VERWEISE}

ANDRADE, Carlos Mauricio Soares de. Pastejo Rotacionado: Tecnologia para Aumentar a Produtividade de Leite e a Longevidade das Pastagens. EMBRAPA - Acre, dez. 2008.

ANUÁRIO LEITE 2019. Sua excelência, o consumidor: novos produtos e novas estratégias da cadeia do leite para ganhar competitividade e conquistar os clientes finais. Brasília, DF: Texto Comunicação Corporativa, 2019. Disponível em: <https://www.infoteca.cnptia.embrapa.br/infoteca/handle/doc/1109959>. Acesso em: 25 mar. 2020.

DEMSKI, Joana Baptista. Desempenho e comportamento de vacas lactantes em pastagens de cultivares de braquiárias. Dissertação apresentada ao Programa de

RC: 99523

Verfügbar in: https://www.nucleodoconhecimento.com.br/veterinariade/umlaufendes-weiden 
Pós-graduação do Instituto de Zootecnia, APTA/SAA. Nova Odessa - SP, 2013. $80 \mathrm{p}$.

FONSECA, Delermando Miranda da; MARTUSCELLO, Janaina Azevedo. Plantas forrageiras. Viçosa, 537p. 2010.

FUKUMOTO, Nelson Massaru; DAMASCENO, Júlio Cesar; DERESZ, Fermino, MARTINS, Carlos Eugênio; CÓSER, Antônio Carlos; SANTOS, Geraldo Tadeu dos. Produção e composição do leite, consumo de matéria seca e taxa de lotação em pastagens de gramíneas tropicais manejadas sob lotação rotacionada. Revista Brasileira de Zootecnia, v. 39, n.7, p. 1548-1557, 2010.

GERON, Luiz Juliano Valério; MEXIA, Alexandre Agostinho; GARCIA Jocilaine; SILVA Marciano Moreira da; ZEOULA, Lúcia Maria. Suplementação concentrada para cordeiros terminados a pasto sobre custo de produção no período da seca. Semina: Ciências Agrárias, Londrina, v. 33, p. 797-808, 2012.

GERON, Luiz Juliano Valério; MOURA, Daiane Caroline; RODRIGUES, Deivison Novaes; PAULA, Edson Júnior Heitor; TRAUTMANN-MACHADO, Raquel Joana; GARCIA, Jocilaine; SCHUMANN, Alline Mariá; SILVA; Dilma Alves. Viabilidade econômica de tourinhos terminados em confinamento alimentados com diferentes teores de caroço de algodão em dietas elaboradas com coprodutos agroindustriais. Semina: Ciências Agrárias, Londrina, v.35, p. 2673-2684, 2014.

GONÇALVES, Carlos Alberto; CAMARÃO, Ari Pinheiro; DUTRA, Saturnino; AZEVEDO, Guilherme Pantoja Calandrini de; MENDONÇA, Denise Castro; SOBRINHO, Carlos de Melo Junior. Produção de leite em pastejo rotacionado intensivo de "Brachiaria brizantha" cv. Marandu sob dois níveis de suplementação concentrado. In: Reunião anual da sociedade brasileira de zootecnia, 40., 2003, Santa Maria. Anais... Santa Maria: Sociedade Brasileira de Zootecnia, 2003

RC: 99523

Verfügbar in: https://www.nucleodoconhecimento.com.br/veterinaria- 
IBGE -Instituto Brasileiro de Geografia e Estatística. [2018]. Pesquisa da pecuária nacional disponível em: <https://sidra.ibge.gov.br/Tabela/74>. Acessado em: 25 mar. 2020.

IPARDES: Instituto Paranaense de Desenvolvimento Econômico e Social. Caracterização sócio econômica da atividade leiteira no Paraná: Sumário executivo. Curitiba: IPARDES, 2009. 29 p.

JANK, Liana; ANDRADE, Carlos Mauricio Soares de; BARBOSA, Rodrigo Amorim; MACEDO, Manuel Claudio Motta; VALERIO, Jose Raul; VERZIGNASSI, Jaqueline Rosemeire; ZIMMER, Ademir Hugo; FERNANDES, Celso Dornelas; SANTOS, Mateus Figueiredo; RESENDE, Rosangela Maria Simeão. O capim-BRS Quênia (Panicum maximum Jacq.) Diversifizierung und Intensivierung der Weiden. Verfügbar in: <https: ainfo.cnptia.embrapa.br/digital/bitstream/item/165106/1/capimbrs-quenia-panicum-maximum-jacq.pdf="'>.</https:> Zugang am: 25 Mär. 2020.

MARION, Jose Carlos; SEGATTI, Sonia. Contabilidade da Pecuária. 9 ed. São Paulo: Atlas, 2010.

MARTIN, Nelson Batista; SERRA, Renata; OLIVEIRA, Marli Dias Mascarenhas; ÂNGELO, José Alberto; OKAWA, Hiroshige. Sistema "CUSTAGRI": sistema integrado de custos agropecuários. São Paulo: IEA/SAA, 1997. p. 1-75.

MARTINEZ, Junior Cesar. Produção de leite a pasto: manejo do pastejo é fundamental para manter a produção. 2009. Disponível em: $<$ http://www.milkpoint.com.br/radartecnico/nutricao/producao-deleite-a-pasto-manejodo-pastejo-e-fundamental-para-manter-aproducao-51710n.aspx>. Acesso em: 27 mai. 2020.

MATSUNAGA, Minoru; BEMELMANS, Paul Frans; TOLEDO, Paulo Edgard Nascimento de; DULLEY, Richard Domingues; HIROSHIGE, Okawa; PEDROSO, Iby

RC: 99523

Verfügbar in: https://www.nucleodoconhecimento.com.br/veterinariade/umlaufendes-weiden 
Arvatti. Metodologia de custo utilizada pelo IEA. Agricultura em São Paulo, v. 23, n. 1, p.123-39, 1976.

OLIVEIRA, Jefferson Soares de. Análise de eficiência e alocação de recursos na produção leiteira do estado do Rio de Janeiro. In: congresso da sociedade brasileira de economia, administração e sociologia rural, 45., 2007. Londrina. Conhecimentos para a agricultura do futuro: Anais... Brasília, DF: SOBER; Londrina: IAPAR: Universidade Estadual de Londrina, 2007.

PEREIRA, Lilian Elgalise Techio. et al. Recomendações para correção e adubação de pastagens tropicais. Pirassununga: Faculdade de Zootecnia e Engenharia de Alimentos da USP, 2018. 56p.

ROSESTOLATO, Lucas Luiz Rocha. et al. Viabilidade econômica comparativa de sistemas tecnológicos da pecuária leiteira no município de Ilha Solteira/SP. Congresso de extensão universitária da UNESP, 8., p. 1-6, 2015. Disponível em: <http://hdl.handle.net/11449/142491>. Acesso em: 28 mai. de 2020.

SEAB - Secretaria de Estado da Agricultura e do Abastecimento. Bovinocultura de leite: leite - $\quad$ produção $2016 / 17 . \quad$ Disponível em:<http://www.agricultura.pr.gov.br/arquivos/File/deral/Prognosticos/2018/leite_201 7_18.pdf>. Acesso em: 25 mar. de 2020.

Gepostet: Juli 2021.

Genehmigt: September 2021.

RC: 99523

Verfügbar in: https://www.nucleodoconhecimento.com.br/veterinaria- 